\title{
Your first foray into clinical research
}

\author{
Alan N. Peiris MD, PhD, FRCP (London), Cathy Lovett RN, MSN, Thomas Tenner PhD
}

At this juncture, you have probably accepted that doing research is an essential step in your development as a physician. What may arise out of need for scholarship may become a lifelong passion. Moreover, passion for a core idea is an essential tool for project completion. Apart from improving job prospects and election to medical honor societies, doing clinical research enables the development of critical thinking skills that may prove lifesaving for your patients and provide a higher level of job satisfaction as a physician. ${ }^{1}$

While the choice of clinical versus basic research is a personal one, clinical research has the added benefit of direct interaction with patients and arguably relates better with ongoing clinical training. The Accreditation Council for Graduate Medical Education (ACGME) expects trainees to participate in research and for residency programs to document such activity.

Although many individuals have had some research experience, either as a medical student or during residency training, writing a research paper as the main contributor remains a daunting task. There are several steps that have to occur when preparing such a paper, so working with a faculty member experienced in medical writing is highly recommended. When choosing a project, consider the following: a focused specific question and time needed for the project.

There are numerous ways in which research

Corresponding author: Alan Peiris MD, PhD, FRCP (London).

Contact Information: Alan.peiris@tttuhsc.edu DOI: $10.12746 /$ swrccc2016.0414.177 questions could arise. Rely on your clinical experience; this remains a valuable resource even for novices. Have you seen any unusual problems or conditions in your clinic or on the hospital service? If so, this would be a good place to start. Begin with discussing the idea, problem, or condition with a senior colleague or faculty member. Working with colleagues will usually make the process much smoother and less daunting. They may have an adjunctive role in your paper but may reciprocate and allow you to make a meaningful contribution to their work. This increases your exposure to ongoing research and may allow additional opportunities to publish. Prior to embarking on a research project, a comprehensive search on PUBMED should be done such that your research venture addresses a novel aspect of a medical issue rather than merely attempting to duplicate well proven findings; a literature search can also help you narrow research questions.

Another option for developing a research question is to consider clinical issues that arise in which the traditional interventions are problematic, ineffective, or costly. Could a low tech tool be developed to address some of these clinical deficiencies? For example, could facial dimensions be a valuable tool in conjunction with a standardized questionnaire to detect obstructive sleep apnea? Is there a way in which facial dimensions can be readily recorded in a standardized and reproducible manner and has it been validated in the literature and used for diagnosis of sleep apnea? It is widely recognized that a receding chin is associated with obstructive sleep apnea, which is a very common and underdiagnosed condition.

Another option when searching for a research question is to question dogma in medical practice. Do some traditional interventions actually achieve the desired goal? For example, large dose steroids are often 
used in Pulmonary Medicine. The deleterious effects on bones of these agents perhaps do not receive the attention they merit. Providers often give about 800 IU of vitamin D3 for bone health in patients with steroid induced osteoporosis. Does this dose actually do anything towards bone health or are much larger doses required? Why do providers readily resort to pharmacologic agents such as bisphosphonates without normalizing a basic building block for bone (Vitamin D)? This type of questioning can be applied to other clinical issues and open up many other interesting issues, including the impact of pharmaceutical companies on physician prescribing. If you want to ease your way into medical writing then a letter to the editor on a topic of personal interest from a recent journal article may be appropriate. Contributing to an editorial along with an experienced faculty member is another option. Research is a process, and time from project initiation to publication can be lengthy; this includes the time for submission and approval from the local institutional review board. It takes time to get data in most cases unless you tap into available data either at the national level, e.g., National Health and Nutrition Examination Survey (NHANES), or mine local data if accessible. Data mining and outcomes research remains a growth area.

There are myriad ways of getting into medical research which can be sculpted to suit your talents and interests. At Texas Tech University Health Sciences Center we are fortunate in having a Clinical Research Institute dedicated to helping faculty and trainees do clinical research. However, this lifelong journey of learning begins with a first step.

Author Affiliation: Alan N. Peiris, Cathy Lovett, and Thomas Tenner work in the Clinical Research Institute at Texas Tech University Health Sciences Center in Lubbock, TX.

Received: $1 / 24 / 2016$

Accepted: 3/27/2016

Published electronically: 04/15/2016

Conflict of Interest Disclosures: None

\section{REFERENCES}

1. Mohr DC, Burgess JF. Job Characteristics and job satisfaction among physicians involved with research in the Veterans Health Administration. Acad Med 2011; 86:938-45 